\title{
Impact of $\mathrm{CHADS}_{2}$ Score on Neurological Severity and Long-Term Outcome in Atrial Fibrillation-Related Ischemic Stroke
}

\author{
Dohoung Kim, ${ }^{a}$ Jong-Won Chung, ${ }^{\text {a }}$ Chi Kyung Kim, ${ }^{a}$ Wi-Sun Ryu, ${ }^{a}$ \\ Eun-Sun Park, ${ }^{a}$ Seung-Hoon Lee, ${ }^{a, b}$ Byung-Woo Yoon ${ }^{a, b}$ \\ ${ }^{a}$ Department of Neurology and ${ }^{\mathrm{b}}$ Clinical Research Center for Stroke, Clinical Research Institute, Seoul National University Hospital, \\ Seoul National University College of Medicine, Seoul, Korea
}

\author{
Received January 22, 2012 \\ Revised March 12, 2012 \\ Accepted March 12, 2012

\section{Correspondence} \\ Seung-Hoon Lee, MD, PhD \\ Department of Neurology, \\ Seoul National University Hospital, \\ Seoul National University \\ College of Medicine, \\ 101 Daehak-ro, Jongno-gu, \\ Seoul 110-744, Korea \\ Tel $+82-2-2072-1014$ \\ Fax +82-2-3672-7553 \\ E-mail sb0516@snu.ac.kr

\section{Correspondence} \\ Byung-Woo Yoon, MD, PhD \\ Department of Neurology, \\ Seoul National University Hospital, \\ Seoul National University \\ College of Medicine, \\ 101 Daehak-ro, Jongno-gu, \\ Seoul 110-744, Korea \\ Tel $+82-2-2072-2875$ \\ Fax +82-2-3673-1990 \\ E-mail bwyoon@snu.ac.kr
}

Background and Purpose The $\mathrm{CHADS}_{2}$ (an acronym for congestive heart failure, hypertension, age $\geq 75$ years, diabetes mellitus, and prior stroke or transient ischemic attack or thromboembolism) score is a widely used system for estimating the risk of stroke in patients with atrial fibrillation. However, how the $\mathrm{CHADS}_{2}$ score is related to stroke severity and outcome in patients with strokes due to atrial fibrillation has not yet been elucidated.

Methods We enrolled patients with atrial fibrillation who visited our stroke center within 7 days after the onset of acute ischemic stroke between October 2002 and September 2008. CHADS 2 scores were categorized into three groups: 0 points, low risk; 1 or 2 points, intermediate risk; and 3-6 points, high risk. Poor neurological state was defined as follows: a National Institutes of Health Stroke Scale (NIHSS) score of $\geq 2$, and a modified Rankin Scale (mRS) score of $\geq 3$ at discharge. Mortality information was ascertained as at December 2008.

Results A cohort of 298 patients with atrial-fibrillation-related stroke was included in this study. A high-risk $\mathrm{CHADS}_{2}$ score at admission was a powerful predictor of poor neurological outcome [for NIHSS: odds ratio (OR), 4.17; 95\% confidence interval (CI), 1.76-9.87; for mRS: OR, 2.97; 95\% CI, 1.23-7.16] after controlling for all possible confounders. In addition, a high-risk $\mathrm{CHADS}_{2}$ score was an independent predictor of all causes of death during the follow-up [hazard ratio (HR), 3.01; 95\% CI, 1.18-7.65] and vascular death (HR, 12.25; 95\% CI, 1.50-99.90).

Conclusions Although the $\mathrm{CHADS}_{2}$ score was originally designed to distinguish patients with a future risk of stroke, our study shows that it may also be used to predict poor neurological outcome after atrial-fibrillation-related stroke.

J Clin Neurol 2012;8:251-258

\section{Introduction}

Atrial fibrillation facilitates embolic stroke due to intracardiac thrombus formation caused by increased turbulent blood flow and congestion, and strategies for stroke prevention are the most important issue in patients with atrial fibrillation. ${ }^{1}$ The Framingham study revealed a fivefold increase in the risk of stroke when atrial fibrillation was present. The average an-

(a) This is an Open Access article distributed under the terms of the Creative Commons Attribution Non-Commercial License (http://creativecommons.org/licenses/by-nc/3.0) which permits unrestricted non-commercial use, distribution, and reproduction in any medium, provided the original work is properly cited. nual rate of ischemic stroke among patients with nonvalvular atrial fibrillation is $5 \%{ }^{2-7}$ The rate of stroke in atrial-fibrillation patients increases with age and the presence of other risk factors such as a prior stroke or transient ischemic attack (TIA), hypertension, diabetes mellitus, and congestive heart failure, ${ }^{8,9}$

Since the clotting factor cascade plays a critical role in thrombus formation, warfarin is highly efficacious, and can inhibit effects of the vitamin-K-dependent clotting factors II, VII, $\mathrm{IX}$, and $\mathrm{X} .{ }^{10}$ Although new anticoagulants are about to be introduced, such as dabigatran ${ }^{11}$ and rivaroxaban, in clinical practice, warfarin has been the only choice for stroke prevention in patients with atrial fibrillation for several decades. However, the levels of warfarin required may vary due to its metabolism 
by the cytochrome $\mathrm{P} 450$ system $^{12}$ and low efficacy or complications caused by high concentrations in the body such as systemic or intracerebral hemorrhage have also been problematic. ${ }^{13}$ The prevalence of atrial fibrillation is reported to be up to $10 \%$ in the aged population, ${ }^{14}$ and simple atrial fibrillation may not be associated with the risk of stroke. ${ }^{15}$ In this context, optimal risk stratification of atrial fibrillation is quite helpful, and the CHADS and $\mathrm{CHADS}_{2}$ (an acronym for congestive heart failure, hypertension, age $\geq 75$ years, diabetes mellitus, and prior stroke or TIA or thromboembolism) scoring systems have been useful for selecting candidate patients who must be treated with warfarin; ${ }^{16-18}$ although its general application rate has not been high.

It is understood that an increased $\mathrm{CHADS}_{2}$ score is strongly associated with the incidence of recurrent stroke. Therefore, a higher $\mathrm{CHADS}_{2}$ score might be associated with more severe strokes in patients with atrial fibrillation, as well as with worse neurological status and worse long-term prognosis due to worsened quality of life. However, this hypothesis has rarely been tested. In the present study, we investigated how the $\mathrm{CHADS}_{2}$ score is related to stroke severity and long-term outcome in patients with stroke due to atrial fibrillation.

\section{Methods}

\section{Study population}

Between October 2002 and September 2008, we enrolled a consecutive series of acute ischemic stroke patients with atrial fibrillation who had been admitted to our stroke unit within 7 days after symptom onset $(n=339)$. Patients with other possible potential causes of stroke were initially excluded from the study population by applying the Trial of Org 10172 in Acute Stroke Treatment criteria. From this initial population, we excluded 17 patients whose diagnosis of stroke was not confirmed due to the lack of brain imaging, including magnetic resonance imaging, and further excluded 24 patients without corresponding brain lesions on their brain images. This resulted in 298 stroke patients with atrial fibrillation finally being enrolled in the current study.

All patients received standard medical therapy during and after hospitalization. Collection of the patients' data and recording of all variables were performed prospectively from the beginning of first enrollment by our organized stroke registry system. This study was approved by the local ethics committee [approval no. H-0911-065-301], and complied with the Declaration of Helsinki. Informed consent to participate was obtained from the enrolled patients or their next of kin.

Demographic characteristics and laboratory data We recorded demographic data (age and gender), conventional risk factors, and important laboratory data for all subjects. Conventional risk factors included hypertension, diabetes, hyperlipidemia, smoking, history of a stroke or TIA, and previous use of antiplatelet or anticoagulant medications. Hypertension was diagnosed if the patients exhibited a systolic blood pressure of $>140 \mathrm{~mm} \mathrm{Hg}$ or a diastolic blood pressure of $>90 \mathrm{~mm} \mathrm{Hg}$ at discharge, or had a previous diagnosis of hypertension and antihypertensive medication use. Diabetes was diagnosed if subjects exhibited a fasting glucose level $\geq 7.0$ $\mathrm{mmol} / \mathrm{L}$ or had a previous diagnosis of diabetes and antidiabetic medication use. A diagnosis of hyperlipidemia was made in patients with a history of using cholesterol-lowering agents or who had a fasting serum total cholesterol level of $>6.0$ $\mathrm{mmol} / \mathrm{L}$ or a low-density lipoprotein cholesterol level of $>4.14$ $\mathrm{mmol} / \mathrm{L}$ at admission. Smoking was coded as positive if the patient was a current smoker or an ex-smoker prior to stroke onset. Body mass index was calculated using height and weight values $\left(\mathrm{kg} / \mathrm{m}^{2}\right)$. Fasting blood samples were drawn within 24 hours of admission, and examined for glucose, lipids, and a standard battery of biochemical and hematological tests.

Atrial fibrillation was diagnosed by standard electrocardiography during hospitalization. $\mathrm{CHADS}_{2}$ scoring was performed using the established scoring system, the components of which items are congestive heart failure (1 point), hypertension (1 point), age of 75 years or older (1 point), diabetes mellitus (1 point), and a prior stroke or TIA (2 points). ${ }^{16,17}$

\section{Neurological severity and mortality}

We assessed each patient's clinical state after ischemic stroke with regard to both neurological severity and mortality. First, neurological severity after the stroke was estimated using the National Institutes of Health Stroke Scale (NIHSS) or the modified Rankin Scale (mRS) at discharge. Second, mortality information was obtained from Statistics Korea, a governmental statistics office in South Korea, as at December 2008, as described previously. ${ }^{19-21}$ The date and cause of death, according to the International Classification of Diseases, 10th Revision, were also recorded..$^{22}$ Mortality data were classified into vascular death and nonvascular death. Vascular death was defined as death caused by stroke, myocardial infarction, heart failure, pulmonary embolism, cardiac arrhythmia, or other definite vascular causes. Nonvascular death was defined as death caused by nonvascular causes such as accidents, cancer, pulmonary causes (e.g., pneumonia or chronic obstructive pulmonary disease), and other miscellaneous causes.

\section{Statistical analysis}

The neurological severity was defined based on the NIHSS or mRS score at discharge. For a severe neurological outcome, we determined the cut-offs as an NIHSS score of 2 or more ${ }^{23}$ 
and an mRS score of 3 or more. ${ }^{24}$ As baseline data, means for continuous variables and proportions for categorical variables were compared using Student's $t$-test, the Mann-Whitney U test, and Pearson's $\chi^{2}$ test, as appropriate, according to dichotomous classifications of NIHSS or mRS scores. We then reclassified the $\mathrm{CHADS}_{2}$ scores as low risk (0 points), intermediate risk ( 1 or 2 points), or high risk ( 3 to 6 points) for comparisons with various variables including neurological severity or mortality. ${ }^{17,18}$ One-way analysis of variance with the Scheffé post-hoc test and the extended Mantel-Haenszel $\chi^{2}$ for trends were performed to compare the distributions of variables according to each grade of $\mathrm{CHADS}_{2}$ score.

We used a multivariate binary logistic regression analysis test to evaluate neurological severity. To determine whether the power of the association between $\mathrm{CHADS}_{2}$ score and severe neurological outcome was independent of the statistical model setting, we constructed two kinds of multivariate analysis model. In Model 1, together with $\mathrm{CHADS}_{2}$ score, we entered demographic data and conventional risk factors as independent variables, while in Model 2 we further entered clinical variables measured during hospitalization and laboratory variables. In terms of long-term mortality, survival rates after stroke were estimated using the Kaplan-Meier product-limit method, and the survival rates of patients according to $\mathrm{CH}$ $\mathrm{ADS}_{2}$ score were compared using the log-rank test. To exam- ine the relationship between grade of $\mathrm{CHADS}_{2}$ score and mortality during the follow-up, Cox proportional-hazards regression analysis was used to calculate the crude and adjusted hazard ratios with $95 \%$ confidence intervals. Two-tailed $p$ values of $<0.05$ were considered significant. Data analysis was performed using SPSS version 17.0 (SPSS Inc., Chicago, IL, USA).

\section{Results}

Of the 298 subjects with atrial-fibrillation-related ischemic stroke, 159 were men (53.4\%) and 139 were women, and they were aged $69.6 \pm 10.2$ years (mean \pm standard deviation). The NIHSS score at discharge was $6.1 \pm 8.2$, and the number of patients with an $\mathrm{mRS}$ score of $\geq 3$ at discharge was 124 (41.6\%).

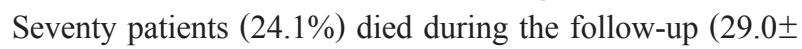
20.8 months), with vascular death being the cause in 37 (13.8\%). According to $\mathrm{CHADS}_{2}$ score, there were 100, 144, and 54 patients in the high-, intermediate-, and low-risk groups, respectively. Baseline characteristics according to the neurological severity are given in Table 1 . Patients with more severe neurological outcomes (NIHSS scores $\geq 2$ or mRS scores $\geq 3$ ) were more likely to be female, to be older, and to have a greater fasting glucose level at admission. High-risk $\mathrm{CHADS}_{2}$ scores were more common among patients with more severe neurological outcomes.

Table 1. Baseline characteristics of the patients

\begin{tabular}{|c|c|c|c|c|c|c|c|}
\hline & \multirow{2}{*}{$\begin{array}{c}\text { All subjects } \\
(n=298)\end{array}$} & \multicolumn{2}{|c|}{ NIHSS } & \multirow[b]{2}{*}{$\begin{array}{c}\mathrm{p} \\
\text { value }\end{array}$} & \multicolumn{2}{|c|}{$\mathrm{mRS}$} & \multirow[b]{2}{*}{$\begin{array}{c}p \\
\text { value }\end{array}$} \\
\hline & & $\begin{array}{c}\text { Mild } \\
(n=1111)\end{array}$ & $\begin{array}{l}\text { Severe } \\
(n=187)\end{array}$ & & $\begin{array}{c}\text { Mild } \\
(n=174)\end{array}$ & $\begin{array}{l}\text { Severe } \\
(n=124)\end{array}$ & \\
\hline Gender, male & $159(53.4 \%)$ & $67(60.4 \%)$ & $92(49.2 \%)$ & 0.06 & $103(59.2 \%)$ & $56(45.2 \%)$ & 0.02 \\
\hline Age over 75 & 103 (34.6\%) & $28(25.2 \%)$ & $75(40.1 \%)$ & 0.01 & $49(28.2 \%)$ & $54(43.5 \%)$ & 0.01 \\
\hline Boby mass index & $23.2 \pm 3.5$ & $23.3 \pm 3.1$ & $23.1 \pm 3.7$ & 0.32 & $23.4 \pm 3.1$ & $22.9 \pm 4.0$ & 0.02 \\
\hline Congestive heart failure & $26(8.7 \%)$ & $6(5.4 \%)$ & $20(10.7 \%)$ & 0.12 & $18(10.3 \%)$ & $8(6.5 \%)$ & 0.24 \\
\hline Hypertension & $197(66.1 \%)$ & $65(58.6 \%)$ & $132(70.6 \%)$ & 0.03 & $109(62.6 \%)$ & $88(71.0 \%)$ & 0.14 \\
\hline Diabetes & $85(28.5 \%)$ & $25(22.5 \%)$ & $60(32.1 \%)$ & 0.08 & $49(28.2 \%)$ & $36(29.0 \%)$ & 0.87 \\
\hline Previous stroke or TIA & $81(27.2 \%)$ & $26(23.4 \%)$ & $55(29.4 \%)$ & 0.26 & $42(24.1 \%)$ & $39(31.5 \%)$ & 0.16 \\
\hline Hyperlipidemia & $56(18.8 \%)$ & $22(19.8 \%)$ & $34(18.2 \%)$ & 0.73 & $29(16.7 \%)$ & $27(21.8 \%)$ & 0.27 \\
\hline Smoking & $75(25.2 \%)$ & $33(29.7 \%)$ & $42(22.5 \%)$ & 0.16 & $52(29.9 \%)$ & $23(18.5 \%)$ & 0.03 \\
\hline Previous antiplatelet use & $120(40.3 \%)$ & $48(43.2 \%)$ & $72(38.5 \%)$ & 0.42 & $76(43.7 \%)$ & $44(35.5 \%)$ & 0.16 \\
\hline Previous anticoagulant use & $62(21.0 \%)$ & $24(21.8 \%)$ & $38(20.5 \%)$ & 0.80 & $36(20.9 \%)$ & $26(21.1 \%)$ & 0.97 \\
\hline Systolic BP at admission & $148 \pm 26$ & $143 \pm 21$ & $151 \pm 29$ & 0.19 & $145 \pm 24$ & $152 \pm 29$ & 0.02 \\
\hline Diastolic BP at admission & $85 \pm 18$ & $84 \pm 14$ & $86 \pm 20$ & 0.49 & $85 \pm 15$ & $86 \pm 21$ & 0.75 \\
\hline Glucose, mmol/L & $6.6 \pm 2.3$ & $6.4 \pm 2.3$ & $6.7 \pm 2.2$ & 0.02 & $6.4 \pm 2.3$ & $6.7 \pm 2.2$ & 0.02 \\
\hline Total cholesterol, mmol/L & $4.4 \pm 0.9$ & $4.4 \pm 0.9$ & $4.5 \pm 0.9$ & 0.52 & $4.4 \pm 0.9$ & $4.5 \pm 1.0$ & 0.15 \\
\hline $\mathrm{CHADS}_{2}$ score & & & & 0.01 & & & 0.03 \\
\hline Low risk & $56(18.1 \%)$ & $28(25.2 \%)$ & $26(13.9 \%)$ & & $36(20.7 \%)$ & $18(14.5 \%)$ & \\
\hline Intermediate risk & 144 (48.3\%) & $57(51.4 \%)$ & $87(46.5 \%)$ & & $90(51.7 \%)$ & $54(43.5 \%)$ & \\
\hline High risk & $100(33.6 \%)$ & $26(23.4 \%)$ & $74(39.6 \%)$ & & $48(27.6 \%)$ & 52 (41.9\%) & \\
\hline
\end{tabular}

Values are means \pm SD or Nos. of patients (percentage). Student's t-test, Mann-Whitney U test, and Pearson's $\chi^{2}$ tests were used. BP: blood pressure, mRS: modified Rankin Scale, NIHSS: National Institutes of Health Stroke Scale. 
The distributions of demographic and clinical characteristics according to $\mathrm{CHADS}_{2}$ score are given in Table 2. The distributions of age, presence of hypertension, diabetes or congestive heart failure, history of a TIA or stroke, blood glucose level, initial systolic blood pressure, and previous use of antiplatelet agents or anticoagulants differed with the $\mathrm{CHADS}_{2}$ score, as revealed by one-way analysis of variance tests and the extended Mantel-Haenszel $\chi^{2}$ for trends $(p<0.05)$. In terms of neurological outcome at discharge, NIHSS scores were significantly higher in the high-risk group, and the distributions of $\mathrm{mRS}$ also differed between the groups. The mortality rate was much higher in the high-risk $\mathrm{CHADS}_{2}$ group, with deaths from all causes and vascular death being 3.8 times (42.9\%) and 9.5 times (51.4\%) more common, respectively, than in the low-risk group (11.4\% and 5.4\%, respectively).

The results of the multivariate logistic regression analyses for neurological severity at discharge are given in Table 3 . When we used the NIHSS score at discharge to represent the neurological outcome, high-risk patients had a greater risk of a severe neurological outcome (adjusted odds ratio, $4.17 ; 95 \%$

Table 2. Distribution of demographic and clinical characteristics according to the $\mathrm{CHADS}_{2}$ score

\begin{tabular}{|c|c|c|c|c|c|}
\hline & \multirow{2}{*}{$\begin{array}{c}\text { All subjects } \\
(n=298)\end{array}$} & \multicolumn{3}{|c|}{$\mathrm{CHADS}_{2}$ score } & \multirow{2}{*}{$\begin{array}{l}p \text { for } \\
\text { trend }\end{array}$} \\
\hline & & Low $(n=54)$ & Intermediate $(n=144)$ & High $(n=100)$ & \\
\hline Gender, male & $159(53.4 \%)$ & $28(51.9 \%)$ & $80(55.6 \%)$ & $51(51.0 \%)$ & 0.76 \\
\hline Age & $69.6 \pm 10.2$ & $62.4 \pm 9.0$ & $70.1 \pm 9.8$ & $72.7 \pm 9.6$ & $<0.001$ \\
\hline Body mass index & $23.2 \pm 3.5$ & $22.5 \pm 3.0$ & $23.6 \pm 3.8$ & $22.9 \pm 3.1$ & 0.09 \\
\hline Hypertension & $197(66.1 \%)$ & $0(0 \%)$ & $107(74.3 \%)$ & $90(90.0 \%)$ & $<0.001$ \\
\hline Diabetes & $85(28.5 \%)$ & $0(0 \%)$ & $36(25.0 \%)$ & $49(49.0 \%)$ & $<0.001$ \\
\hline Hypercholesterolemia & $56(18.8 \%)$ & $7(13.0 \%)$ & $25(17.4 \%)$ & $24(24.0 \%)$ & 0.08 \\
\hline Congestive heart failure & $26(8.7 \%)$ & $0(0 \%)$ & $11(7.6 \%)$ & $15(15.0 \%)$ & 0.001 \\
\hline Previous stroke or TIA & $81(27.2 \%)$ & $0(0 \%)$ & $7(4.9 \%)$ & $74(74.0 \%)$ & $<0.001$ \\
\hline Smoking & 75 (25.2\%) & $11(20.4 \%)$ & $37(25.7 \%)$ & $27(27.0 \%)$ & 0.40 \\
\hline Glucose, $\mathrm{mmol} / \mathrm{L}$ & $6.6 \pm 2.3$ & $5.5 \pm 1.2$ & $6.7 \pm 2.4$ & $6.9 \pm 2.3$ & $<0.001$ \\
\hline Total cholesterol, $\mathrm{mmol} / \mathrm{L}$ & $4.4 \pm 0.9$ & $4.4 \pm 0.9$ & $4.4 \pm 1.0$ & $4.5 \pm 0.9$ & 0.58 \\
\hline Systolic blood pressure, $\mathrm{mm} \mathrm{Hg}$ & $148 \pm 26$ & $140 \pm 21$ & $148 \pm 25$ & $151 \pm 29$ & 0.03 \\
\hline Diastolic blood pressure, $\mathrm{mm} \mathrm{Hg}$ & $85 \pm 18$ & $83 \pm 16$ & $85 \pm 15$ & $87 \pm 22$ & 0.42 \\
\hline Antiplatelet use & $120(40.3 \%)$ & $13(24.1 \%)$ & $63(43.8 \%)$ & $44(44.0 \%)$ & 0.04 \\
\hline Anticoagulation use & $62(21.0 \%)$ & $11(20.8 \%)$ & 20 (14.0\%) & $31(31.3 \%)$ & 0.04 \\
\hline NIHSS score & $6.1 \pm 8.2$ & $4.2 \pm 6.3$ & $5.5 \pm 8.0$ & $7.9 \pm 9.0$ & 0.01 \\
\hline mRS & & & & & 0.01 \\
\hline Mild (<3) & $174(58.4 \%)$ & $36(66.7 \%)$ & $90(62.5 \%)$ & $48(48.0 \%)$ & \\
\hline Severe $(\geq 3)$ & $124(41.6 \%)$ & 18 (33.3\%) & $54(37.5 \%)$ & $52(52.0 \%)$ & \\
\hline All-cause death & $70(24.1 \%)$ & $8(11.4 \%)$ & $32(45.7 \%)$ & $30(42.9 \%)$ & 0.02 \\
\hline Vascular death & $37(13.8 \%)$ & $2(5.4 \%)$ & $16(43.2 \%)$ & $19(51.4 \%)$ & 0.01 \\
\hline
\end{tabular}

Values are means \pm SD or Nos. of patients (percentage). One-way analysis of variance tests or Mantel-Haenszel $\chi^{2}$ tests were used. mRS: modified Rankin Scale, NIHSS: National Institutes of Health Stroke Scale, TIA: transient ischemic attack.

Table 3. Adjusted odd ratios of NIHSS and mRS

\begin{tabular}{|c|c|c|c|c|}
\hline & \multicolumn{2}{|l|}{ NIHSS } & \multicolumn{2}{|l|}{ mRS } \\
\hline & Adjusted OR (95\% CI) & $p$ value & Adjusted OR (95\% CI) & $p$ value \\
\hline \multicolumn{5}{|l|}{ Model 1* } \\
\hline Low risk & Reference & & Reference & \\
\hline Intermediate risk & $1.76(0.91-3.39)$ & 0.09 & $1.31(0.66-2.60)$ & 0.44 \\
\hline High risk & $3.61(1.73-7.56)$ & 0.001 & $2.64(1.27-5.49)$ & 0.01 \\
\hline \multicolumn{5}{|l|}{ Model $2^{+}$} \\
\hline Low risk & Reference & & Reference & \\
\hline Intermediate risk & $2.19(1.01-4.73)$ & 0.05 & $1.59(0.70-3.63)$ & 0.27 \\
\hline High risk & $4.17(1.76-9.87)$ & 0.001 & $2.97(1.23-7.16)$ & 0.02 \\
\hline
\end{tabular}

${ }^{*}$ Model 1: $\mathrm{CHADS}_{2}$ score+gender, smoking, hypercholesterolemia, previous use of antiplatelet or anticoagulant, ${ }^{\dagger} \mathrm{Model} 2$ : $\mathrm{CHADS}_{2}$ score+gender, smoking, hypercholesterolemia, previous use of antiplatelet or anticoagulant, BMI, intrahospital treatment of IV, IA thrombolytics, or heparinization, systolic and diastolic blood pressure, serum glucose, total cholesterol.

BMI: body mass index, mRS: modified Rankin Scale, NIHSS: National Institutes of Health Stroke Scale, OR: odds ratio. 
confidence interval 1.76-9.87; reference, low-risk patients) in both Model 1 (adjusted for epidemiological and historical variables) and Model 2 (further adjusted for in-hospital and laboratory variables). In addition, similar results with slightly stronger associations were obtained when we used mRS scores to represent the neurological outcome.

Survival curves are illustrated in Fig. 1. The overall survival among all of the patients differed significantly between the $\mathrm{CHADS}_{2}$ groups, as determined by the log-rank test $(p=0.004)$. Survival from vascular death also differed significantly ( $p=$ $0.004)$, whereas that from nonvascular death did not $(p=0.18)$. The crude and adjusted hazard ratios of each $\mathrm{CHADS}_{2}$ group by Cox proportional-hazards regression analyses of death from all causes are listed in Table 4; the low-risk group was used as a reference. The rate of death from all causes was related to $\mathrm{CHADS}_{2}$ score in a stepwise fashion ( $p$ for trend= 0.006). After adjustment, high-risk $\mathrm{CHADS}_{2}$ scores significantly increased the risk of death from all causes. This trend between $\mathrm{CHADS}_{2}$ groups and death from all causes was also significant ( $p$ for trend=0.04). The results for vascular death were similar, with the high-risk $\mathrm{CHADS}_{2}$ score being an inde-
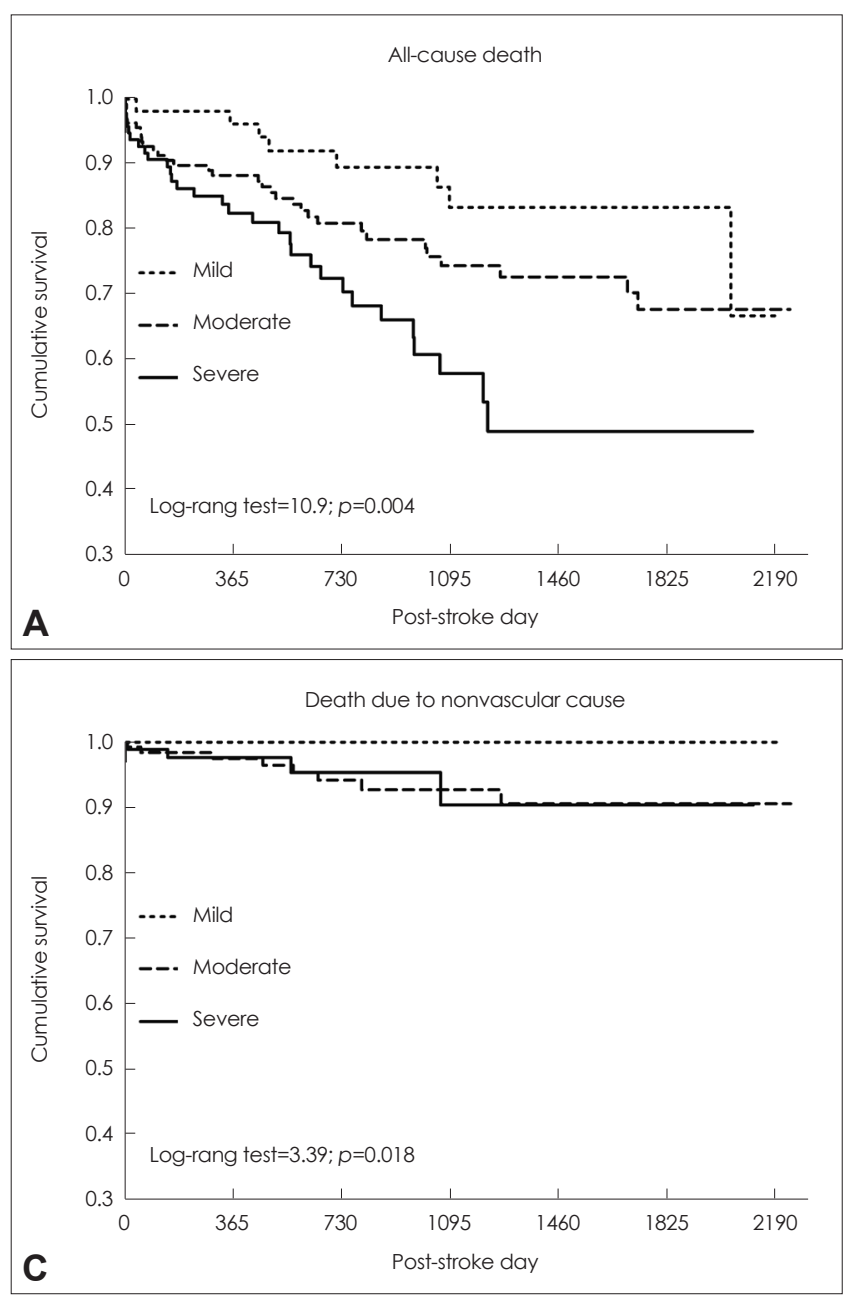

pendent risk factor and there being a significant dose-response trend $(p$ for trend $=0.006$ ).

\section{Discussion}

We found that high-risk $\mathrm{CHADS}_{2}$ scores were associated with a worse neurological outcome at discharge and an increased long-term mortality, especially due to vascular causes; it is noteworthy that the associations remained significant after adjustment for in-hospital and laboratory profiles. Since $\mathrm{CH}$ $\mathrm{ADS}_{2}$ scores can be calculated in individuals even before a stroke event, these results might be useful for estimating future risk of stroke and for designing stroke-prevention strategies for patients with atrial fibrillation.

The $\mathrm{CHADS}_{2}$ scoring system is an algorithm for estimating the risk for embolic stroke in atrial-fibrillation patients, and so higher scores might be associated with an increased amount of thrombus in the heart, which could obstruct largersized arteries in the brain and cause more severe neurological damage. ${ }^{25,26}$ Specifically, a decreased ejection fraction and increased turbulent flow in congestive heart failure will more

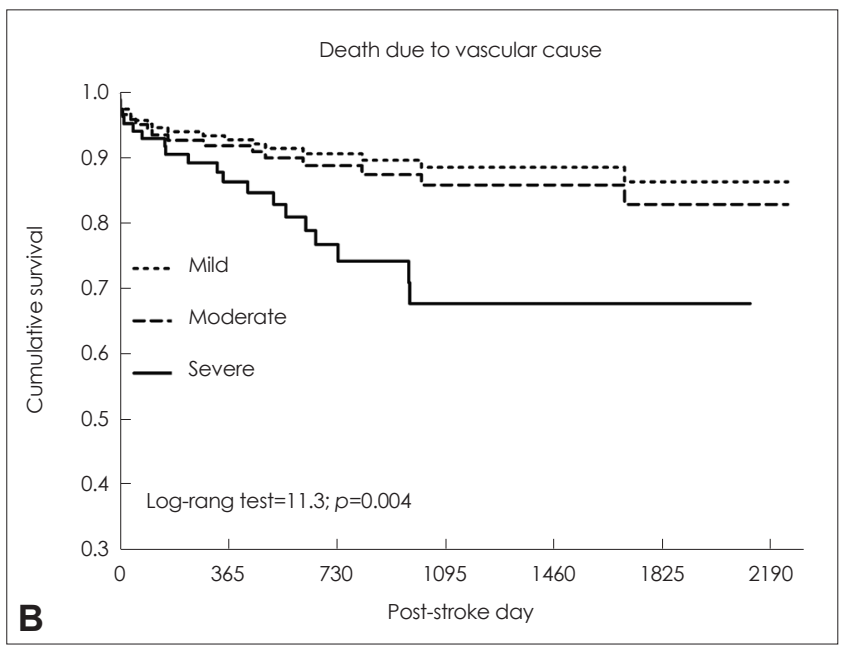

Fig. 1. Kaplan-Meier curves of all-cause (A), vascular (B), and nonvascular (C) deaths. 
Table 4. Unadjusted and adjusted hazard ratios of all-cause death and death due to vascular cause

\begin{tabular}{|c|c|c|c|c|}
\hline & \multicolumn{2}{|c|}{ All-cause death } & \multicolumn{2}{|c|}{ Death due to vascular cause } \\
\hline & Unadjusted HR (95\% CI) & Adjusted HR (95\% Cl) & Unadjusted HR (95\% Cl) & Adjusted HR (95\% CI) \\
\hline \multicolumn{5}{|l|}{$\mathrm{CHADS}_{2}$ score } \\
\hline Low risk & \multicolumn{2}{|c|}{ Reference } & \multicolumn{2}{|c|}{ Reference } \\
\hline Intermediate risk & $1.79(0.83-3.89)$ & $1.62(0.65-4.01)$ & $3.26(0.75-14.2)$ & $3.86(0.47-31.8)$ \\
\hline High risk & $3.21(1.46-7.05)$ & $3.01(1.18-7.65)$ & $6.95(1.61-30.0)$ & $12.25(1.50-99.9)$ \\
\hline$p$ for trend & 0.006 & 0.04 & 0.008 & 0.006 \\
\hline Gender, male & & $0.68(0.35-1.32)$ & & $0.38(0.15-1.00)$ \\
\hline Smoking & & $1.59(0.79-3.21)$ & & $1.62(0.60-4.39)$ \\
\hline Hypercholesterolemia & & $0.70(0.32-1.53)$ & & $0.78(0.30-2.05)$ \\
\hline Antiplatelet use & & $0.86(0.47-1.57)$ & & $0.64(0.27-1.50)$ \\
\hline Anticoagulation use & & $0.87(0.42-1.80)$ & & $0.50(0.18-1.43)$ \\
\hline Body mass index & & $0.91(0.83-0.99)$ & & $0.96(0.85-1.08)$ \\
\hline IV thrombolytics & & $1.47(0.64-3.37)$ & & $1.18(0.34-4.09)$ \\
\hline IA thrombolytics & & $2.05(0.75-5.58)$ & & $0.70(0.09-5.39)$ \\
\hline Heparinization & & $0.86(0.48-1.54)$ & & $0.81(0.37-1.80)$ \\
\hline Systolic blood pressure & & $1.01(0.99-1.02)$ & & $1.01(0.99-1.03)$ \\
\hline Diastolic blood pressure & & $0.99(0.97-1.02)$ & & $0.99(0.96-1.04)$ \\
\hline Glucose & & $1.01(1.00-1.01)$ & & $1.00(1.00-1.01)$ \\
\hline Total cholesterol & & $1.01(1.00-1.01)$ & & 1.01 (1.00-1.02) \\
\hline
\end{tabular}

Adjusted HR were adjusted for gender, smoking, hypercholesterolemia, previous use of antiplatelet or anticoagulant, BMI, intrahospital treatment of IV, IA thrombolytics, or heparinization, systolic and diastolic blood pressure, serum glucose, and total cholesterol. BMI: body mass index, HR: hazards ratio.

frequently give rise to a larger thrombus. In addition, hypertension, diabetes, and advanced age have been reported to be associated with increased blood coagulability. ${ }^{27-29}$ Biochemical markers would reflect the coagulability more precisely in patients with atrial fibrillation. ${ }^{14}$

Gustafsson et al. ${ }^{30}$ found that patients with atrial fibrillation had higher concentrations of von Willebrand factor, fibrinogen, D-dimer, and factor VIII. In addition, Lip et al. ${ }^{31}$ reported a significant correlation between fibrinogen and Ddimer in patients with atrial fibrillation. It was also previously reported that prothrombic markers are related with each component of the $\mathrm{CHADS}_{2}$ score. There was a correlation between hypertension and the level of von Willebrand factor and Eselectin. ${ }^{32}$ Furthermore, insulin resistance has been associated with plasminogen activator inhibitor-1, von Willebrand factor, coagulation factors VII, VIII, and XII, and fibrinogen. However, it is difficult to apply these biomarkers at the primary hospital level due to poor cost-effectiveness; our strategy using the $\mathrm{CHADS}_{2}$ scoring system might be easier to apply.

One of the strengths of this study is the excellent validity of the mortality information. In Korea, all residents must register their identity information, including the births or deaths of family members, in the Korean Resident Registration System, which has been the only governmental system of the Family Registration Act since 1962. If a family member dies, the other family members must report his or her death with a medical certificate issued by a hospital within 15 days of the death. The death certificate should include information on the exact identification, time, and place of death, and the medical cause of death according to the International Classification of Diseases, 10th Revision. This system is mandatory in Korea, and the statistical results obtained using data from this system have been highly reliable.

In the present study, long-term mortality was clearly improved in patients with high-risk $\mathrm{CHADS}_{2}$ scores. This relationship may be explained by the two assumptions. First, the patients with high-risk $\mathrm{CHADS}_{2}$ scores had multiple vascular risk factors (e.g., hypertension and diabetes). Accordingly, they might have had a higher risk of experiencing vascular events after the onset of stroke, and these multiple recurrent vascular events might aggravate their medical and neurological status, and ultimately increase the risk of mortality during the follow-up. This assumption may be related to the association only being significant for vascular death. Second, we can suggest an explanation of the results related to neurological outcome. The neurological outcomes at discharge (as measured by NIHSS and mRS scores) were more severe in patients with high-risk $\mathrm{CHADS}_{2}$ scores. After discharge, the quality of life of the patients with severe neurological disability must have been poor due to the decreased daily activity and an increased frequency of medical or neurological complications. From a statistical point of view, severe neurological out- 
come appears to act as a mediator for increased mortality.

There are also some important caveats to this study. First, as mentioned in the Methods section, this study was not designed prior to patient enrollment, although a prospective and consecutive registry system was used, including a variety of demographic, epidemiologic, clinical, and laboratory variables, and all of the components of the $\mathrm{CHADS}_{2}$ scoring system were included in the system. However, we acknowledge that the generalizability of our findings may be limited because this was a single-center study and the most of the included subjects were Korean. Second, we estimated stroke severity using NIHSS and mRS scores "at discharge" instead of the more common time of 3 months after onset. However, a recent report on this issue has indicated that neurological scores at discharge sufficiently represent the final neurological outcome of patients. ${ }^{33}$ Furthermore, we condensed our data using mortality information. Third, all of the patients included in this study had atrial fibrillation, but in some cases their strokes were caused by other mechanisms such as small-vessel occlusion due to hypertension. We attempted to minimize this limitation by applying the standardized Trial of Org 10172 in Acute Stroke Treatment criteria. Finally, we did not obtain information on compliance to medication course and International Normalized Ratio levels during the follow-up. This limitation could be overcome in a prospectively designed study.

In conclusion, we found that high-risk $\mathrm{CHADS}_{2}$ scores were associated with an aggravated neurological outcome and increased long-term mortality in stroke patients with atrial fibrillation. It might be a natural assumption that the addition of vascular risk factors leads to the occurrence of vascular diseases. Nevertheless, it would be difficult to standardize and estimate the validity of the additive effects of risk factors cannot be easily conducted, and applying an established, validated scoring system is more comprehensive and has a wider potential for application. We believe that our results may be useful for predicting the potential risk of stroke in patients with atrial fibrillation.

\section{Conflicts of Interest}

The authors have no financial conflicts of interest.

\section{Acknowledgements}

This study was supported by a grant from the Korea Health 21 R\&D Project, Ministry of Health and Welfare, Republic of Korea [no. A100331].

\section{REFERENCES}

1. Fatkin D, Kelly RP, Feneley MP. Relations between left atrial appendage blood flow velocity, spontaneous echocardiographic contrast and thromboembolic risk in vivo. J Am Coll Cardiol 1994;23:961-969.

2. Flegel KM, Shipley MJ, Rose G. Risk of stroke in non-rheumatic atrial fibrillation. Lancet 1987;1:526-529.

3. Wolf PA, Abbott RD, Kannel WB. Atrial fibrillation: a major contribu- tor to stroke in the elderly. The Framingham Study. Arch Intern Med 1987; 147:1561-1564.

4. Wolf PA, Abbott RD, Kannel WB. Atrial fibrillation as an independent risk factor for stroke: the Framingham Study. Stroke 1991;22:983-988.

5. Risk factors for stroke and efficacy of antithrombotic therapy in atrial fibrillation. Analysis of pooled data from five randomized controlled trials. Arch Intern Med 1994;154:1449-1457.

6. Krahn AD, Manfreda J, Tate RB, Mathewson FA, Cuddy TE. The natural history of atrial fibrillation: incidence, risk factors, and prognosis in the Manitoba Follow-Up Study. Am J Med 1995;98:476-484.

7. Lévy S, Maarek M, Coumel P, Guize L, Lekieffre J, Medvedowsky JL, et al. Characterization of different subsets of atrial fibrillation in general practice in France: the ALFA study. The College of French Cardiologists. Circulation 1999;99:3028-3035.

8. Hart RG, Pearce LA, Aguilar MI. Meta-analysis: antithrombotic therapy to prevent stroke in patients who have nonvalvular atrial fibrillation. Ann Intern Med 2007;146:857-867.

9. Stroke Risk in Atrial Fibrillation Working Group. Independent predictors of stroke in patients with atrial fibrillation: a systematic review. Neurology 2007;69:546-554.

10. Mackman N. Triggers, targets and treatments for thrombosis. Nature 2008;451:914-918.

11. Connolly SJ, Ezekowitz MD, Yusuf S, Eikelboom J, Oldgren J, Parekh A, et al. Dabigatran versus warfarin in patients with atrial fibrillation. N Engl J Med 2009;361:1139-1151.

12. Krynetskiy E, McDonnell P. Building individualized medicine: prevention of adverse reactions to warfarin therapy. J Pharmacol Exp Ther 2007;322:427-434.

13. Palareti G, Leali N, Coccheri S, Poggi M, Manotti C, D’Angelo A, et al. Bleeding complications of oral anticoagulant treatment: an inception-cohort, prospective collaborative study (ISCOAT). Italian Study on Complications of Oral Anticoagulant Therapy. Lancet 1996;348: 423-428.

14. Feng D, D’Agostino RB, Silbershatz H, Lipinska I, Massaro J, Levy D, et al. Hemostatic state and atrial fibrillation (the Framingham Offspring Study). Am J Cardiol 2001;87:168-171.

15. Kopecky SL, Gersh BJ, McGoon MD, Whisnant JP, Holmes DR Jr, Ilstrup DM, et al. The natural history of lone atrial fibrillation. A population-based study over three decades. N Engl J Med 1987;317:669-674.

16. Gage BF, Waterman AD, Shannon W, Boechler M, Rich MW, Radford MJ. Validation of clinical classification schemes for predicting stroke: results from the National Registry of Atrial Fibrillation. JAMA 2001; 285:2864-2870.

17. Gage BF, van Walraven C, Pearce L, Hart RG, Koudstaal PJ, Boode $\mathrm{BS}$, et al. Selecting patients with atrial fibrillation for anticoagulation: stroke risk stratification in patients taking aspirin. Circulation 2004; 110:2287-2292.

18. Fang MC, Go AS, Chang Y, Borowsky L, Pomernacki NK, Singer DE, et al. Comparison of risk stratification schemes to predict thromboembolism in people with nonvalvular atrial fibrillation. $\mathrm{J} \mathrm{Am} \mathrm{Coll} \mathrm{Car-}$ diol 2008;51:810-815.

19. Lee SH, Kim BJ, Ryu WS, Kim CK, Kim N, Park BJ, et al. White matter lesions and poor outcome after intracerebral hemorrhage: a nationwide cohort study. Neurology 2010;74:1502-1510.

20. Ryu WS, Lee SH, Kim CK, Kim BJ, Yoon BW. Effects of low serum triglyceride on stroke mortality: a prospective follow-up study. Atherosclerosis 2010;212:299-304.

21. Kim BJ, Lee SH, Ryu WS, Kim CK, Lee J, Yoon BW. Paradoxical longevity in obese patients with intracerebral hemorrhage. Neurology 2011;76:567-573.

22. Manual of the International Statistical Classification of Diseases, Injuries, and Causes of Death. 10th revision. Geneva, Switzerland: World Health Organization, 1992.

23. Young FB, Weir CJ, Lees KR; GAIN International Trial Steering Committee and Investigators. Comparison of the National Institutes of He- 
alth Stroke Scale with disability outcome measures in acute stroke trials. Stroke 2005;36:2187-2192.

24. Weisscher N, Vermeulen M, Roos YB, de Haan RJ. What should be defined as good outcome in stroke trials; a modified Rankin score of 0-1 or 0-2? J Neurol 2008;255:867-874.

25. Barreto AD, Albright KC, Hallevi H, Grotta JC, Noser EA, Khaja AM, et al. Thrombus burden is associated with clinical outcome after intraarterial therapy for acute ischemic stroke. Stroke 2008;39:3231-3235.

26. Puetz V, Dzialowski I, Hill MD, Subramaniam S, Sylaja PN, Krol A, et al. Intracranial thrombus extent predicts clinical outcome, final infarct size and hemorrhagic transformation in ischemic stroke: the clot burden score. Int J Stroke 2008;3:230-236.

27. Lip GY, Blann AD. Does hypertension confer a prothrombotic state? Virchow's triad revisited. Circulation 2000;101:218-220.

28. Tofler GH, Massaro J, Levy D, Mittleman M, Sutherland P, Lipinska I, et al. Relation of the prothrombotic state to increasing age (from the Framingham Offspring Study). Am J Cardiol 2005;96:1280-1283.
29. Grant PJ. Diabetes mellitus as a prothrombotic condition. $J$ Intern Med 2007;262:157-172.

30. Gustafsson C, Blombäck M, Britton M, Hamsten A, Svensson J. Coagulation factors and the increased risk of stroke in nonvalvular atrial fibrillation. Stroke 1990;21:47-51.

31. Lip GY, Lowe GD, Rumley A, Dunn FG. Fibrinogen and fibrin D-dimer levels in paroxysmal atrial fibrillation: evidence for intermediate elevated levels of intravascular thrombogenesis. Am Heart J 1996; 131:724-730.

32. Blann AD, Waite MA. von Willebrand factor and soluble E-selectin in hypertension: influence of treatment and value in predicting the progression of atherosclerosis. Coron Artery Dis 1996;7:143-147.

33. Blinzler C, Breuer L, Huttner HB, Schellinger PD, Schwab S, Köhrmann M. Characteristics and outcome of patients with early complete neurological recovery after thrombolysis for acute ischemic stroke. $\mathrm{Ce}$ rebrovasc Dis 2011;31:185-190. 